\title{
Abelhas sem Ferrão (Hymenoptera: Apidae, Meliponini) da Estação Ecológica de Água Limpa, Cataguases-MG, Brasil
}

\author{
Hugo de Azevedo Werneck ${ }^{\bowtie}$, Georgina Maria Faria-Mucci ${ }^{2}$
}

1. Universidade Federal de Viçosa/ Faculdades Integradas de Cataguases-FIC UNIS, e-mail: beehugo@gmail.com (Autor para correspondência ${ }^{\varpi}$ ). 2. Faculdades Integradas de Cataguases-FIC UNIS/ Universidade do Estado de Minas Gerais-UEMG, Ubá, e-mail: gfariamucci@gmail.com.

\section{EntomoBrasilis 7 (2): 164-166 (2014)}

Resumo. No presente estudo realizou-se um levantamento de ninhos de abelhas Meliponini na Estação Ecológica de Água Limpa (EEAL), no município de Cataguases, Minas Gerais. Foram encontrados 21 ninhos pertencentes a nove espécies: Friesella schrottkyi (Friese), Melipona bicolor Lepeletier, Plebeia sp. 1, Plebeia sp. 2, Tetragona clavipes (Fabricius), Tetragona quadrangula (Fabricius), Tetragonisca angustula (Latreille), Trigona hyalinata (Lepeletier), Trigona spinipes (Fabricius). Vinte dos 21 ninhos foram encontrados em substrato arbóreo. A fauna de Meliponini observada na EEAL é representada por um baixo número de espécies. Embora a vegetação local tenha potencial para oferecer substratos para nidificação e recursos florais, a baixa riqueza de espécies pode estar relacionada com o fato da EEAL ser um remanescente florestal isolado.

Palavras-Chave: Mata Atlântica; Insecta; Meliponini; Substrato de nidificação.

\section{Stingless Bees of the Estação Ecológica de Água Limpa, Cataguases-MG, Brazil}

Abstract. This study carried out a survey of nests of stingless bees present in the Estação Ecológica de Água Limpa (EEAL) in the municipality of Cataguases, Minas Gerais. We found twenty-one nests belonging to nine species: Friesella schrottkyi (Friese), Melipona bicolor Lepeletier, Plebeia sp. 1, Plebeia sp. 2, Tetragona clavipes (Fabricius), Tetragona quadrangula (Fabricius), Tetragonisca angustula (Latreille), Trigona hyalinata (Lepeletier), Trigona spinipes (Fabricius). Twenty of the 21 nests were found in arboreal substrate. The fauna of stingless bees observed in EEAL is represented by a low number of species. Although the local vegetation has the potential to provide substrates for nesting and floral resources, the low species richness may be related to the fact that the EEAL is an isolated forest remnant.

Keywords: Atlantic forest; Insecta; Meliponini; Nesting substrate.

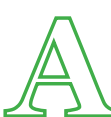
s abelhas popularmente conhecidas como indígenas sem ferrão (Nogueira-Neto 1997) pertencem à tribo Meliponini (Moure et al. 2007). Essas abelhas possuem o ferrão atrofiado e são incapazes de ferroar, o que deu origem ao nome popular. A tribo Meliponini compreende cerca de 60 gêneros distribuídos pelas regiões tropicais e subtropicais do mundo (MiCHENER 2007).

Todas as espécies de Meliponini são eusociais. O comportamento eusocial é caracterizado pela divisão de trabalho entre os membros da colônia, sobreposição de gerações e uma clara divisão de castas, sendo as operárias as não-reprodutivas, e a rainha a casta reprodutiva (Wilson 1971; MichENER 1974, 2007; HöLLDOBLER \& WILSON 2009).

A estrutura e localização dos ninhos de Meliponini são bastante diversificadas. Diferentes tipos de substratos para nidificação podem ser utilizados como ocos em árvores ou ninhos expostos construídos entre os galhos, mourões de cerca, cupinzeiros e cavidades no solo (Roubik 1989, 2006; Michener 2007). Algumas espécies são abundantes nas áreas com influência antrópica, como Tetragonisca angustula (Latreille), e Nannotrigona testaceicornis (Lepeletier), podendo construir seus ninhos em postes e caixas de energia ou cavidades em muros e paredes.

As abelhas, de um modo geral, são totalmente dependentes dos recursos florais para a sua alimentação, sendo responsáveis pela polinização de cerca de 40 a 90\% das espécies vegetais conforme o ecossistema, sendo de extrema importância para a manutenção da biodiversidade (KerR et al. 1996). Os Meliponini visitam várias espécies de plantas para a coleta de pólen, néctar e resinas. Uma característica importante do forrageamento dessas abelhas é o fato delas possuírem constância floral, o que faz com que elas visitem repetidamente uma única espécie de planta durante seu período de floração, o que as torna eficientes polinizadores (Roubık 1989). Além disso, diversas espécies de Meliponini são utilizadas para a polinização de plantas cultivadas (Drucker 2004; WeSTERKAMP \& GOTTSBERGER 2006).

Portanto, as abelhas sem ferrão são peça chave na manutenção de diversos ecossistemas em que ocorrem, sendo importante o desenvolvimento de estratégias para a sua preservação. Uma das maiores ameaças para os Meliponini é o desmatamento, visto que muitas espécies necessitam de árvores com ocos para nidificação, além disso, o uso abusivo de defensivos agrícolas em plantações é outra ameaça constante (KERR et al, 1996).

O objetivo do presente estudo foi verificar a composição da fauna de abelhas sem ferrão da Estação Ecológica de Água Limpa (EEAL) através do levantamento de seus ninhos.

A EEAL está localizada nas coordenadas $21^{\circ} 22$ 'S e $42^{\circ} 42^{\prime} \mathrm{W}$, com uma área total de 70 ha no município de Cataguases-MG ao leste da Zona da Mata de Minas Gerais. O clima da região,

Agência(s) de Financiamento: FAPEMIG 
T segundo a classificação de Köppen, varia do tipo Cwa, tropical úmido a Aw, semi-úmido de verões quentes (Коттек et al. 2006). A vegetação predominante é de Mata Atlântica do tipo Estacional Semidecídua. A área da EEAL até o início do século passado era uma grande fazenda de café. Foi transformada em Horto Florestal no ano de 1916 e reflorestada com espécies nativas e exóticas (Werneck \& CARVAlHo 2008). Para realizar a busca de ninhos, foram elaborados transectos que cobriram áreas dentro e fora das trilhas. Foram realizadas visitas mensais ao campo entre os meses de março de 2006 a abril de 2007. A altura de cada ninho foi medida para determinar a distribuição vertical. Dez indivíduos de cada ninho encontrado foram coletados para identificação. O material testemunho está depositado no Museu Regional de Entomologia do Departamento de Entomologia da Universidade Federal de Viçosa (MEUFV).

Foram encontrados 21 ninhos pertencentes a nove espécies distribuídas em cinco gêneros (Tabela 1). T. angustula (Figura 1A) foi a espécie mais abundante, representando $42,8 \%$ do total amostrado. As espécies menos abundantes, das quais foi encontrado apenas um ninho foram: Friesella schrottkyi (Friese), Plebeia sp. 1, Plebeia sp. 2, e Tetragona quadrangula (Lepeletier). Todas as espécies amostradas na EEAL apresentam ampla distribuição geográfica (Tabela 1) ocorrendo em diferentes ecossistemas.

Com relação ao substrato, 20 dos 21 ninhos foram encontrados em substrato arbóreo, apenas um ninho de T. angustula foi construído no barranco. A distribuição vertical dos ninhos variou entre o e $15 \mathrm{~m}$ de altura do solo, sendo Trigona hyalinata (Lepeletier) (Figura 1B) a espécie que nidificou mais alto, e Melipona bicolor Lepeletier (Figura 1C, D) a espécie que nidificou mais próximo do solo.

Tabela 1. Espécies de Meliponini encontradas na Estação ecológica de Água Limpa, quantidade de ninhos, substratos utilizados para nidificação e distribuição geográfica. ${ }^{*}$ Segundo Moure et al. (2007)

\begin{tabular}{|c|c|c|c|}
\hline Espécie & No de ninhos & Substrato & Distribuição Geográfica* \\
\hline Friesella schrottkyi (Friese) & 1 & arbóreo & Brasil (ES, MG, PR, SP). \\
\hline Melipona bicolor Lepeletier & 2 & arbóreo & Brasil (BA, ES, MG, RJ, SP). \\
\hline Plebeia sp.1 & 1 & arbóreo & - \\
\hline Plebeia sp. 2 & 1 & arbóreo & - \\
\hline Tetragona clavipes (Fabricius) & 2 & arbóreo & $\begin{array}{l}\text { Bolívia, Colômbia, Guiana, Paraguai, Peru, Suriname e Brasil } \\
\text { (AC, AP, AM, BA, ES, GO, MA, MT, MS, MG, PA, PR, PI, RJ, } \\
\text { RS, SC, SP). }\end{array}$ \\
\hline Tetragona quadrangula (Lepeletier) & 1 & arbóreo & Brasil (GO, MA, MT, PA, MG, PA, SP, TO). \\
\hline Tetragonisca angustula (Latreille) & 9 & $\begin{array}{l}\text { arbóreo, } \\
\text { barranco }\end{array}$ & $\begin{array}{l}\text { México, Bolívia, Brasil (praticamente em todo o território } \\
\text { nacional), Colômbia, Costa Rica, Equador, Guatemala, } \\
\text { Guiana, Honduras, Nicarágua, Panamá, Peru, Suriname e } \\
\text { Venezuela. }\end{array}$ \\
\hline Trigona hyalinata (Lepeletier) & 2 & arbóreo & $\begin{array}{l}\text { Bolívia, Paraguai, Brasil (BA, DF, GO, MA, MT, MS, MG, } \\
\text { PA, PI, SP, TO) }\end{array}$ \\
\hline Trigona spinipes (Fabricius) & 2 & arbóreo & $\begin{array}{l}\text { Argentina, Brasil (praticamente em todo o território } \\
\text { nacional), Colômbia, Guiana, Paraguai e Peru. }\end{array}$ \\
\hline
\end{tabular}

A destruição dos remanescentes florestais na região de Cataguases tem ameaçado as populações de abelhas sem ferrão, principalmente as do gênero Melipona. A espécie $M$. bicolor é uma abelha muito sensível aos impactos ambientais, geralmente não saindo de seu ninho se houver perturbações nas redondezas. Elas nidificam em ocos de árvores, frequentemente na base dos troncos e muitas vezes suas entradas passam despercebidas (Nogueira-NeTo 1997).

O estudo da composição da fauna dessas abelhas em remanescentes florestais como a EEAL é de extrema importância para a preservação da flora local bem como para o manejo e criação de espécies com fins econômicos, tais como polinização de culturas.

Embora a vegetação local tenha potencial para oferecer substratos para nidificação e recursos florais, a baixa riqueza de espécies pode estar relacionada com o fato da EEAL ser um remanescente florestal isolado.

Portanto, ressalta-se a necessidade de outros estudos, como coletas diretamente nas flores, estudos dos sistemas de polinização de plantas relacionadas, comportamento de para aprovisionamento dos ninhos através de análises de pólen coletados por essas abelhas.

\section{AGRADECIMENTOS}

Os autores agradecem às Faculdades Integradas de Cataguases e à Universidade Federal de Viçosa pelo apoio logístico e à FAPEMIG pelo apoio financeiro.

\section{REFERÊNCIAS}

Drucker, A.G., 2004. Economic valuation of bee pollination services: implications for farm management and policy, p. 125-134. In: Freitas, B.M \& J.O. Pereira (Eds). Solitary bees: Conservation, Rearing and Management for Pollination. Fortaleza, Imprensa Universitária, 285p.

Hölldobler, B. \& E.O. Wilson, 2009. The Superorganism: The beauty, Elegance and Strangeness of Insect Societies, $1^{\text {st }}$ Ed. Line drawings by Margaret C. Nelson, 522p.

Kerr, W.E., G.A. Carvalho \& V. Nascimento, 1996. A Abelha uruçu: Biologia, Manejo e Conservação. Belo Horizonte, Acangau, 144p.

Kottek, M., J. Grieser, C. Beck, B. Rudolf \& F. Rubel, 2006. World map of the Köppen-Geiger climate classification updated. Meteorologische Zeitschrift, 15: 259-263.

Michener, C.D., 1974. The social behavior of the bees: A comparative study. Belknap Press of Harvard University Press, 404p.

Michener, C.D., 2007. The bees of the World, $2^{\text {nd }}$ Ed. Baltimore, D.C, John Hopkins, 953p.

Moure, J.S., D. Urban \& G.A.R. Melo, 2007. Catalogue of bees 

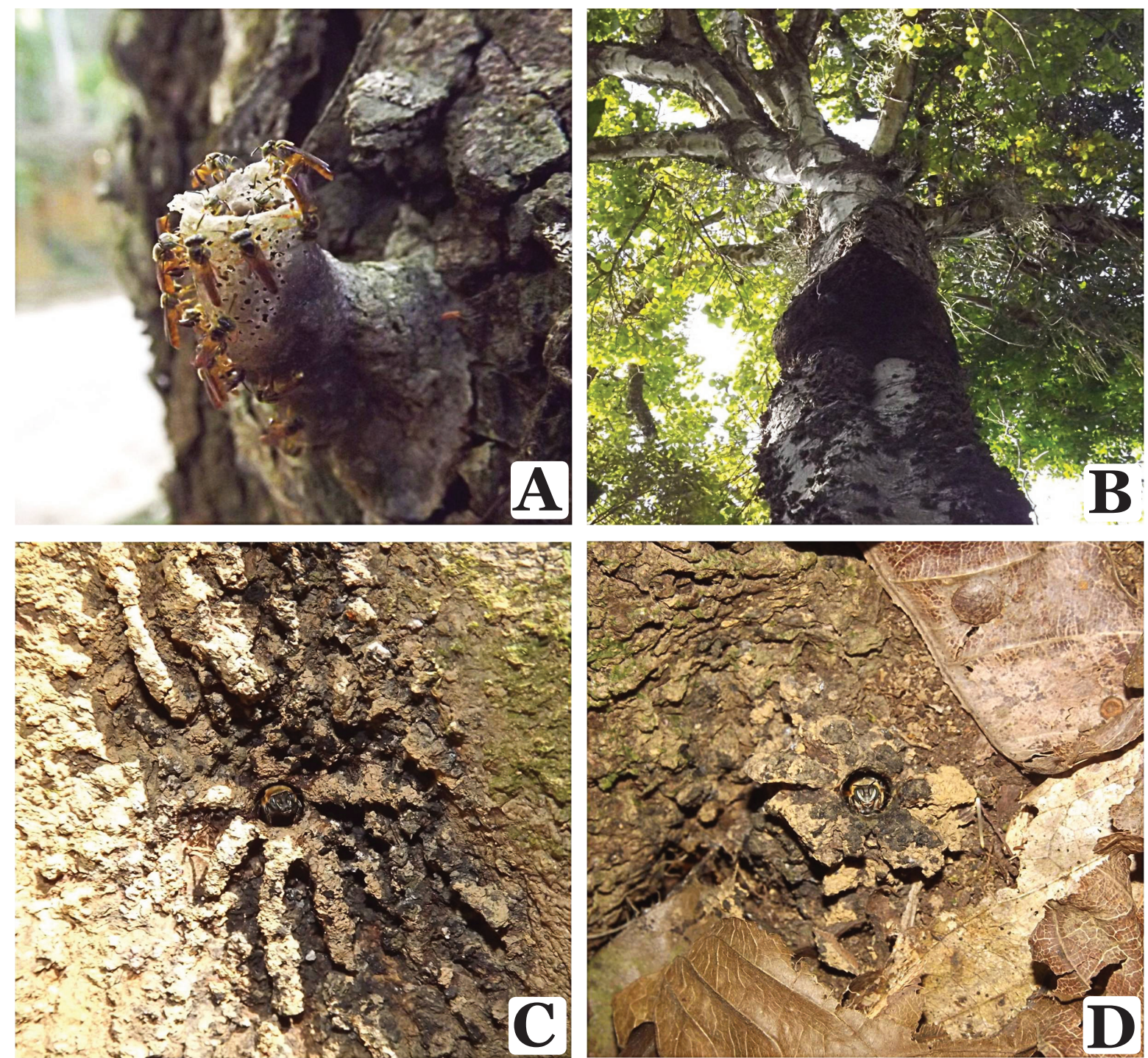

Figura 1. Ninhos de abelhas Meliponini encontrados na Estação Ecológica de Água Limpa, Cataguases, Minas Gerais. A. Tetragonisca angustula; B. Trigona hyalinata; C. Melipona bicolor; D. Melipona bicolor. Fotos: H.A. Werneck

(Hymenoptera, Apoidea) in the Neotropical Region. Curitiba, Sociedade Brasileira de Entomologia, 1058p.

Nogueira-Neto, P., 1997. Vida e Criação de Abelhas indígenas sem Ferrão. São Paulo, Nogueirapis, 446p.

Roubik, D.W., 1989. Ecology and natural history of tropical bees. Cambridge University Press, 514p.

Roubik, D.W., 2006. Stingless bees nesting biology. Apidologie, 37: 124-143.

Werneck, H.A. \& A.M. Carvalho, 2008. Estação Ecológica de Água Limpa - Aspectos Históricos, Culturais e Importância Ambiental para o Município de Cataguases-MG/Brasil. p. 1214. In: V Simpósio de Meio Ambiente, Viçosa-MG, CBCN, 195p.
Westerkamp, C. \& G. Gottsberger, 2006. The costly crop pollination crisis. p. 57-62. In: Kevan, P.G \& V.L. ImperatrizFonseca (Eds.). Pollinating Bees: The Conservation Link Between Agriculture and Nature, 336p.

Wilson, E.O., 1971. The insect Societies. The Belknap Press of Harvard University Press, Cambridge, 420p.

\section{Recebido em: 13/12/2013}

Aceito em: 20/03/2014

Como citar este artigo:

Werneck, H.A. \& G.M. Faria-Mucci, 2014. Abelhas sem Ferrão (Hymenoptera: Apidae, Meliponini) da Estação Ecológica de Água Limpa, CataguasesMG, Brasil. EntomoBrasilis, 7 (2): 164-166.

Acessível em: doi:10.12741/ebrasilis.v7i2.408
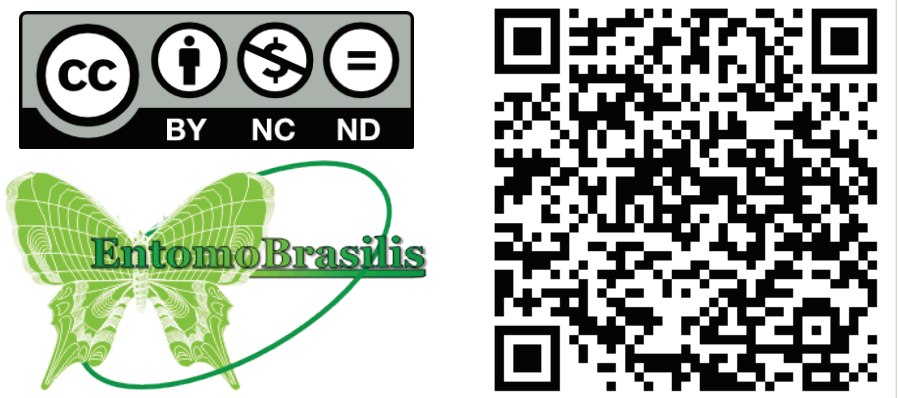